Al-Azhar Bull. Sci. Vol. 18, No. 1 (June.): pp. 103-114, 2007.

\title{
SYNTHESIS OF SOME NEW BENZOFURAN DERIVATIVES OF EXPECTED BIOLOGICAL ACTIVITY
}

\section{S. A. HESSEIN}

Chemistry Department, Faculty of Science (Girl's), Al-Azhar University, Cairo, Egypt

\begin{abstract}
Treatment of 1-[6-hydroxy-4-methoxybenzofuran-5-yl]ethanone (1) with cinnamaldehyde gave 1-[6-hydroxy-4-methoxybenzofuran-5-yl]-5-phenylpenta-2,4-diene-1-one (2) which reacted with formaline and 4-chloroniline to give benzofuran derivative (3) according to Mannich reaction. Interaction of $\mathbf{2}$ with $20 \%$ of sulphuric acid, malononitrile, ethylcyano acetate, phenyl hydrazine and guanidine hydrochloride afforded the corresponding furo[3,2-g]chromon-5-one (4), pyridine, pyrazole and pyrimidine derivatives (5-8). Also, compound 8 reacted with phosphorusoxychloride in dimethyl formamide and p-flurobenzylidenemalononitrile to give formyl and pyrimidopyrimidene derivatives $(\mathbf{1 0}, \mathbf{1 2})$.
\end{abstract}

\section{Introduction}

It is well known that benzofuran derivatives show marked biological activity ${ }^{(1-6)}$. Also, derivatives of pyridine, pyrimidine and pyrazol show a varity of pharmacological effects ${ }^{(7,8)}$. Thus, the aim of the present work is synthesis pyridine, pyrimidine and pyrazole containing benzofuranyl moiety to investigate their potential activity.

\section{Result and discussion}

Reaction of 1-[6-hydroxy-4-methoxybenzofuran-5-yl]ethanone (1) with cinnamaldehyde led to form 1-[6-hydroxy-4-methoxybenzofuran-5-yl]-5phenylpenta-2,4-diene-1-one (2) Scheme (1). Compound 2 was established by correct analytical and spectral data. The mass spectrum afforded a molecular ion peak at $\mathrm{m} / \mathrm{z} 320\left[\mathrm{M}^{+}, 50 \%\right]$ with a base peak at 190 and the following observed peaks at 277 (20.2\%), 230 (16.3\%), 164 (38.04\%), 148 (20.6\%) and $117(16.3 \%)$ which compatible with molecular formula $\mathrm{C}_{20} \mathrm{H}_{16} \mathrm{O}_{4}$ Chart (1).

According to Mannich reaction 2 was reacted with 4-chloroaniline and formaline to yield Mannich base (3) Scheme (1). The mass spectrum of 3 exhibited a molecular ion peak $\mathrm{M}^{+}$at $\mathrm{m} / \mathrm{z} 459$ (2.8\%), corresponding to molecular formula $\mathrm{C}_{27} \mathrm{H}_{22} \mathrm{NO}_{4} \mathrm{Cl}$ and this was in agreement with previous work $^{(9-12)}$. 
When hydroxychalcone 2 was refluxed with $20 \%$ sulphuric acid, cyclization occurred to form furo[3,2-g]chromon-5-one (4a-4b) or the possible isomers Scheme (1). The IR spectrum of compound 4 revealed the presence of carbonyl group of $\gamma$ pyrone at $1620 \mathrm{~cm}^{-1}$, and this analogy with previous work ${ }^{(5,13)}$.

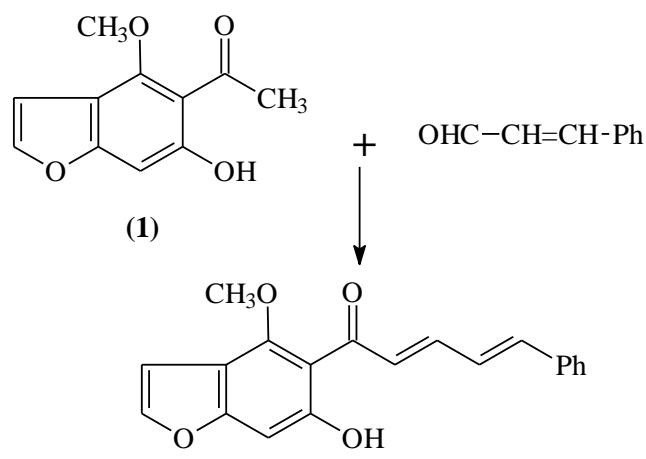

(2)

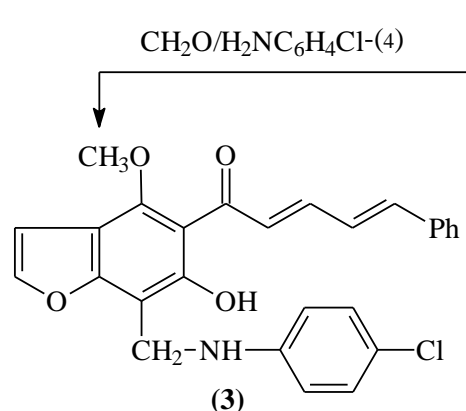

$20 \% \mathrm{H}_{2} \mathrm{SO}_{4}$

(3)<smiles>COc1c2c(cc3occc13)OC(/C=C/c1ccccc1)C=C2O</smiles>

(Scheme 1)

(4b)

Treatment of 2 with malononitrile led to the formation of pyridin-3-carbonitrile or the possible isomer (5a-5b). The reaction can proceeded via chalcone (2) which reacted instantaneously with malononitrile through Michael reaction, then cyclization occurred via elimination of molecule of water and dehydrogenation Scheme (2). The assigned structure was in agreement with analytical and spectral data where IR spectrum exhibited bands at $2194(\mathrm{CN}), 3049,3190\left(\mathrm{NH}_{2}\right)$ and 3340 (OH) groups. While ${ }^{1} \mathrm{H}-\mathrm{NMR}$ of 5 (DMSO) showed signals at $\delta 4.00-4.19(\mathrm{~m}, 5 \mathrm{H}$, 
$\left.\mathrm{OCH}_{3}+\mathrm{NH}_{2}\right), 6.7$ ( , 1H, H-3 furan moiety), 6.72-7.82 (m, 10H, 1H, H-5 pyridine + $\mathrm{Ar}-\mathrm{H}+2 \mathrm{H}$, olefinic $+\mathrm{H}-2$ furan moiety $)$ and at $11.98(, 1 \mathrm{H}, \mathrm{OH}$, exchangeable with $\left.\mathrm{D}_{2} \mathrm{O}\right)$.

Similarly 2 reacted with ethylcyanoacetate to produce pyridin-3-ethyl carboxylate (6) Scheme (2). IR spectrum afforded bands at $1743(\mathrm{C}=\mathrm{O}), 3028$ \& $3236\left(\mathrm{NH}_{2}\right)$ and at $3286(\mathrm{OH})$ groups and this was analogy with previous work ${ }^{(14)}$.

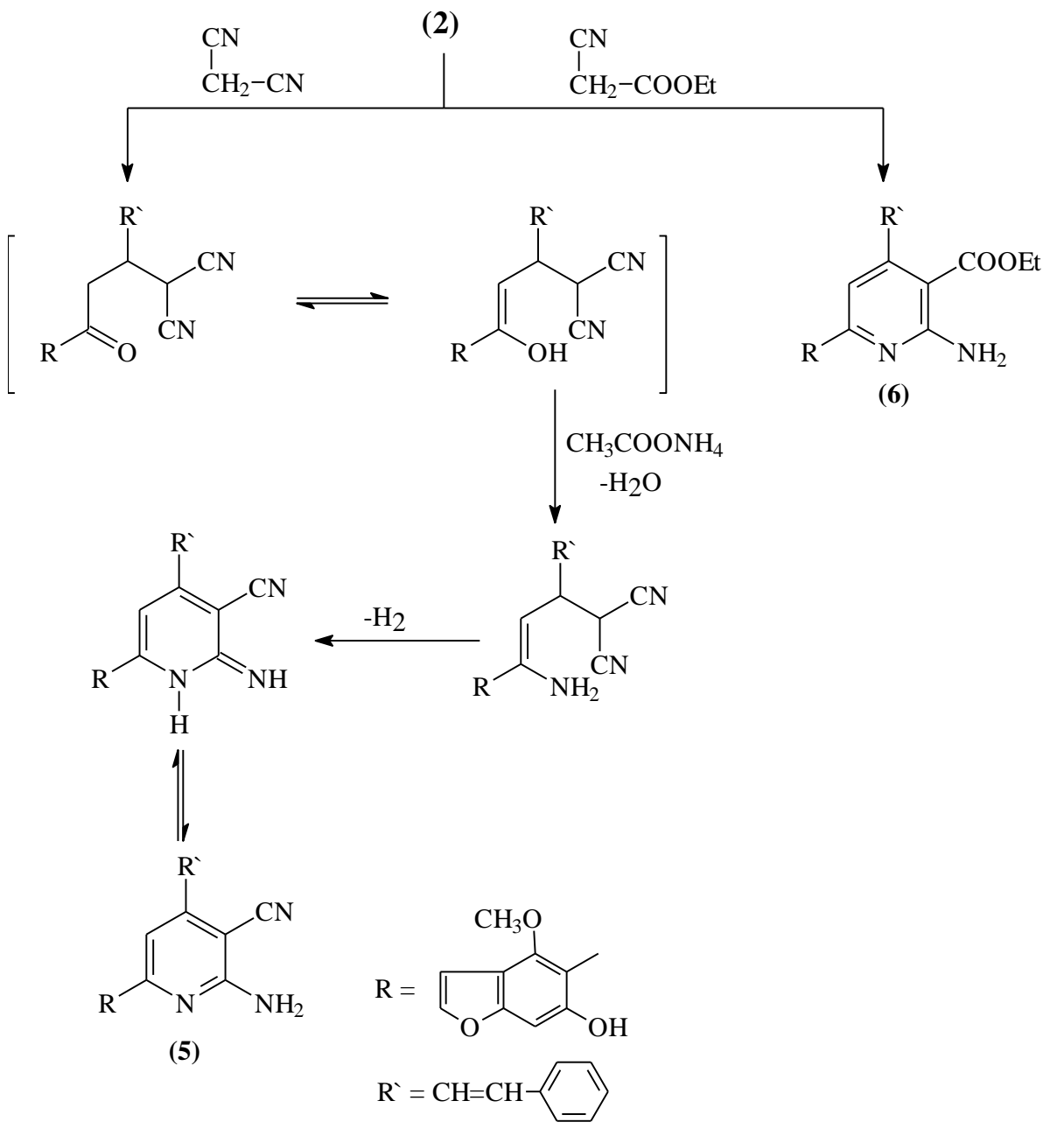

(S cheme 2) 
Compound 2 was reacted with phenylhydrazine to form pyrazole derivative (7) scheme (3). Structure 7 was established by correct analytical and spectral data where IR showed disappearance of carbonyl group found in the parent compound and revealed bands at 1601,3313 for $(\mathrm{C}=\mathrm{N})$ and $(\mathrm{OH})$ groups, respectively.

Furthermore, the reaction of $\mathbf{2}$ towards binucleophilic reagent was investigated. Thus, interaction of $\mathbf{2}$ with guanidine hydrochloride in the presence of potassium hydroxide resulted pyrimidine derivative or its possible isomer (8a-8b) Scheme (3). The reaction was proceeding via Michael addition followed intramolecular cyclization followed by water elimination. IR spectrum of $\mathbf{8}$ showed the disappearance of carbonyl group which found in the parent compound and showed bands at $3124 \& 3164$ for $\mathrm{NH}_{2}$ and 3413 for $\mathrm{OH}$ groups.

In addition, treatment of $\mathbf{8}$ with phosphorusoxychloride in dimethylformamide, the formyl derivative (10) was obtained via vilsmeier reaction, Scheme (3). The proposed structure 10 was based on elemental analysis and IR spectrum which revealed the lack of NH function group and the presence of C-H aldehyde at 2866 \& 2927 and $\mathrm{OH}$ group at 3327. The mass spectrum exhibited a molecular ion peak at $\mathrm{m} / \mathrm{z} 415$ which corresponding to the molecular formula $\mathrm{C}_{23} \mathrm{H}_{17} \mathrm{~N}_{3} \mathrm{O}_{5}$.

Further, compound $\mathbf{8}$ reacted with p-flurobenzylidene malononitrile in the presence of catalytic amount of piperidine to afford pyrimido-pyrimidine derivative (12), via Michael adduct (11) followed by intramolecular cyclization, Scheme (3). The structure of compound $\mathbf{1 2}$ was esablished by elemental analysis and IR spectrum showed the absorption bands at $2190 \mathrm{~cm}^{-1}(\mathrm{C} \equiv \mathrm{N}), 3124 \& 3192\left(\mathrm{NH}_{2}\right)$ and at $3397(\mathrm{OH})$ groups. 


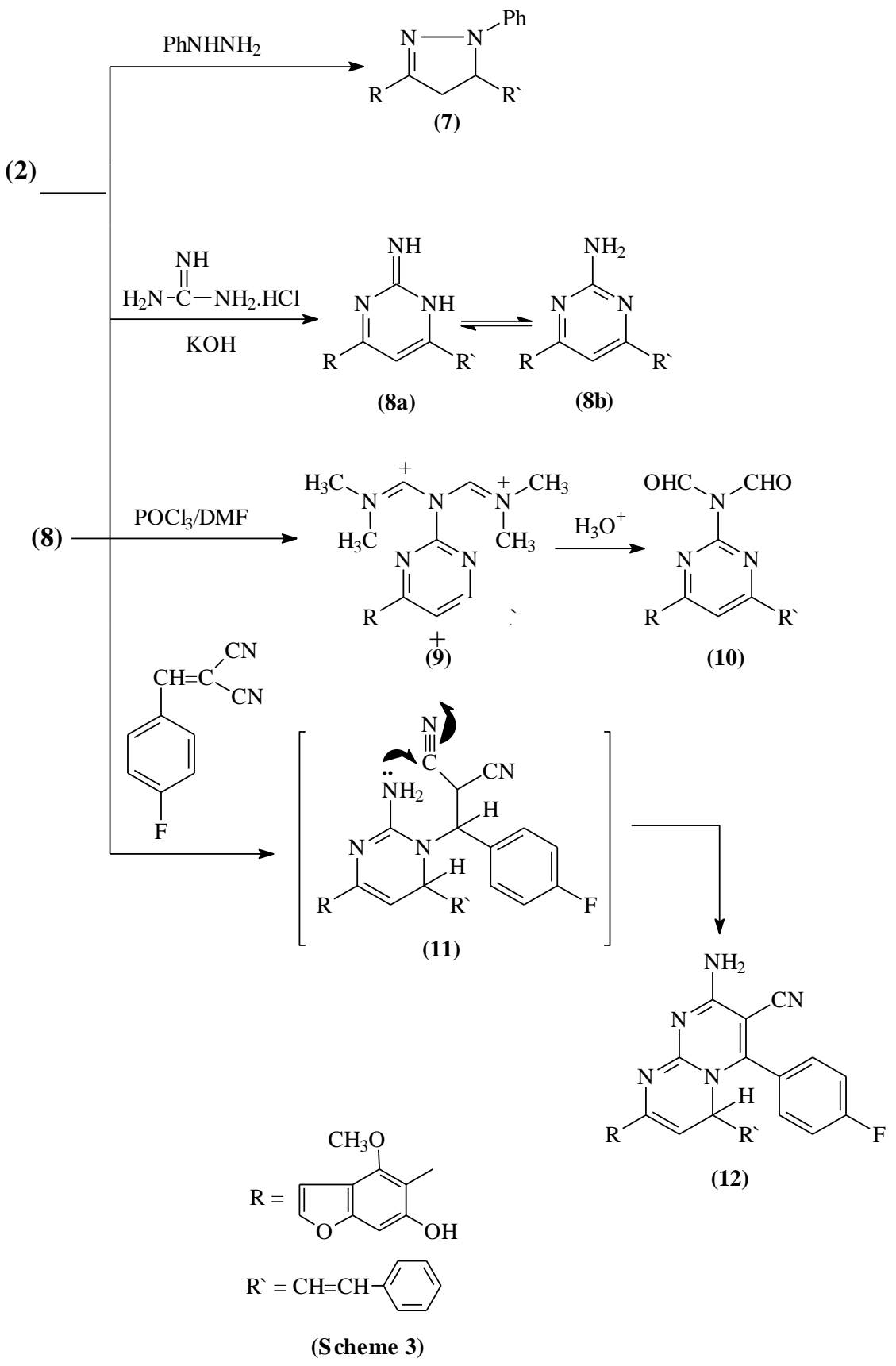


<smiles>C/C=C/C(=O)c1c(O)cc2occc2c1OC</smiles>

(2)

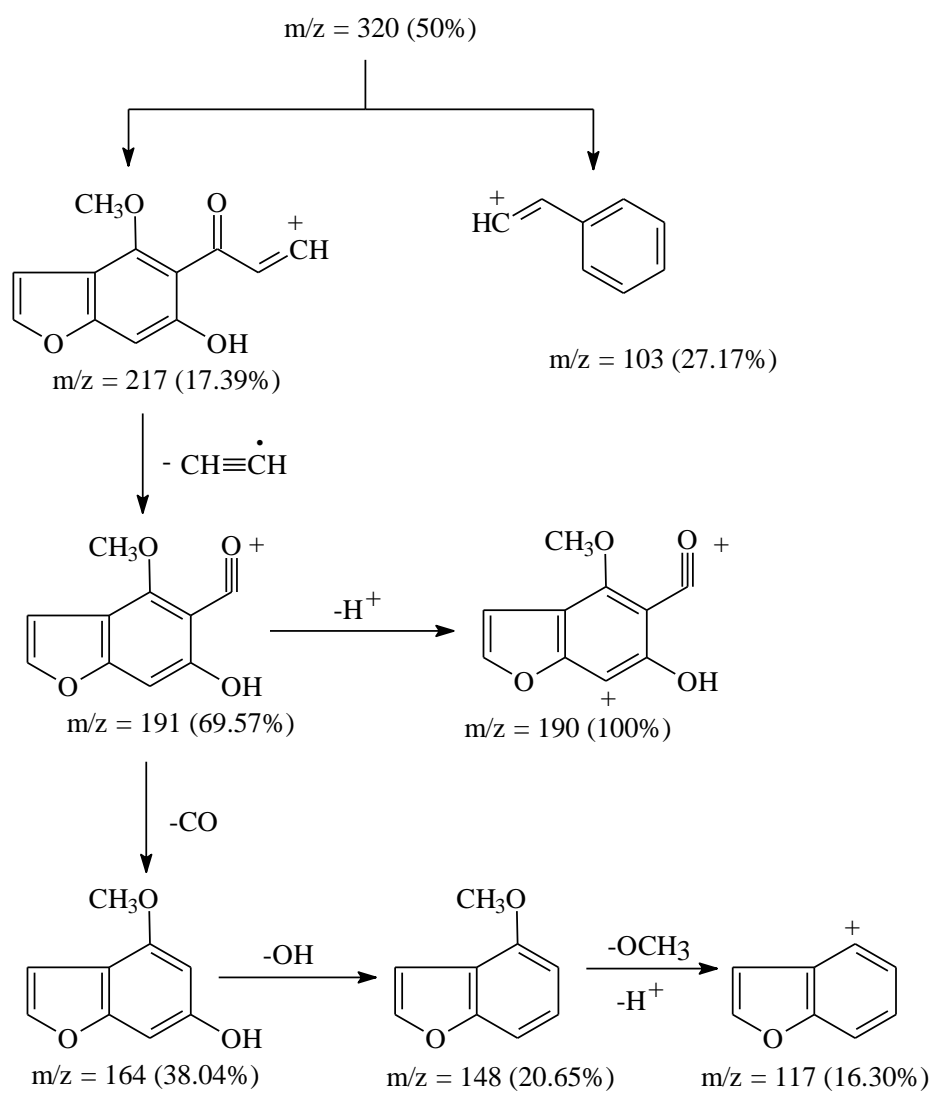

(Chart 1)

\section{Antimicrobial activity of some new benzofuran derivatives:-}

The standardized disc-agar diffusion method (Bauer-Kirby 1966) ${ }^{(15)}$ was followed to determine the activity of the synthesized compounds against the sensitive organisms Staphlococcus aureus (ATCC 25923) and Streptococcus pyogenes (ATCC 19615) as Gram positive bacteria, Pseudomonas fluorescens (S 97) and Pseudomonas phaseolicola (GSPB 2828) as Gram- negative bacteria and the fungi Fusarium oxysporum and Aspergillus fumigatus. 
The broad spectrum antibiotic chloramphencol was used as standard antibacterial reference and cyclohexamide was used as standard antifungal reference.

The tested compounds were dissolved in dimethyl formamide [(DMF) which have no inhibition activity] to get concentrations of $2 \mathrm{mg} / \mathrm{ml}$ and $1 \mathrm{mg} / \mathrm{ml}$. The test was contain infusion of $200 \mathrm{~g}$ potatoes, $6 \mathrm{~g}$ dextrose agar.

Uniform size filter paper disks (3 disks per compound) were impregnated by equal volume $(10 \mu \mathrm{l})$ from the specific concentration of dissolved tested compounds and carefully placed on inoculated agar surface.

After incubation for $36 \mathrm{~h}$ at $27^{\circ} \mathrm{C}$ in the case of bacteria and for $48 \mathrm{~h}$ at $24^{\circ} \mathrm{C}$ in the case of fungi inhibition of the organisms which evidenced by clear zone surround each disk was measured and used to calculate mean of inhibition zones, (Table 2).

The antimicrobial investigation revealed that the compound (2) showed slight activity against gram positive, gram negative bacteria and fungi, compound (4) showed slight activity against gram negative bacteria, compound (5) was completely in active against bacteria strain but high activity against fungi. Compound (7) afforded slight activity against gram positive and gram negative bacteria and completely in active against fungi.

In addition compound (8) showed intermediate activity against gram negative bacteria and slight activity against fungi, while compound $\mathbf{1 0}$ showed high activity against gram positive and gram negative bacteria. This may be due to the presence of pyrimidine moiety.

\section{Experimental}

All melting points were uncorrected. The IR spectra were recorded on pye unicam sp 11100 spectrophotometer. ${ }^{1} \mathrm{H}-\mathrm{NMR}$ spectra were recorded in $\mathrm{CDCl}_{3}$ or in DMSO on a varian 90, $200 \mathrm{MHz}$, spectrometer. Mass spectra were preformed by a shimadzu Gc-MS-QP 100 Ex (shimadzu, Japan). Elemental analysis were carried out by the Microanalytical Research Center, Faculty of Science, Cairo University.

1-[6-hydroxy-4-methoxy benzofuran-5-yl]-5-phenylpenta-2,4-dien-1-one (2)

To a solution of 1 (3.2 gm, $0.01 \mathrm{~mol})$ and cinamaldehyde $(0.01 \mathrm{~mol})$ in ethanol $(30 \mathrm{ml})$ was added $10 \%$ alcoholic sodium hydroxide $(5 \mathrm{ml})$ and the reaction mixture 
was stirred at room temperature for 30 mint. The reaction mixture was acidified with hydrochloric acid and the resulting solid was washed with water and crystallized from ethanol to give $\mathbf{2}$ (Table 1).

\section{1-[7-(4-chlorophenyl)aminomethyl-6-hydroxy-4-methoxybenzo-furan-5 yl]phenylpenta-2,4-dien-1-one (3)}

To a solution of compound 2 (3.2 gm, $0.01 \mathrm{~mol})$ in ethanol $(30 \mathrm{ml})$, 4chloroaniline (1.3 gm, 0.01 mole) and $\mathrm{CH}_{2} \mathrm{O} 40 \%$ formaldehyde $(0.015 \mathrm{~mol})$ were added with stirring for $30 \mathrm{~min}$. and cooling between $0-5^{\circ} \mathrm{c}$. The reaction mixture was left for one hour, the solid so formed was collected and crystallized from acetone to form 3, Table (1). MS. $459\left(\mathrm{M}^{+}, 2 \%\right)$ with a base peak at 89 and the following observed peaks at 416 (1.21\%), 369 (1.9\%), 311 (65.6\%), 239 (4\%), 177 (12.1\%) and $13367.9 \%$ ).

\section{6,7-dihydro-4-methoxy-7-styrylfuro[3,2-g]chromon-5-one (4)}

A solution of $2(3.2 \mathrm{gm}, 0.01 \mathrm{~mol})$ in ethanol $(20 \mathrm{ml})$ and concentrated sulfuric acid $(2 \mathrm{ml}$ ) was refluxed for about $4 \mathrm{hrs}$. Evaporate the solvent and the resulting solid was crystallized from n-hexane to give $\mathbf{4}$ which gave no colour reaction with ferric chloride solution. ${ }^{1} \mathrm{H}$ NMR (DMSO) $\delta 3.96 \mathrm{ppm}\left(1 \mathrm{~s}, 3 \mathrm{H}, \mathrm{OCH}_{3}\right), 6.69-8.007$ $(\mathrm{m}, 10 \mathrm{H}, \mathrm{Ar}-\mathrm{H}+2=\mathrm{CH}+\mathrm{H}-2$ chromon $+\mathrm{H}-3$ furan $)(\mathrm{J}=2.02 \mathrm{~Hz}), \delta 9.06(\mathrm{~d}, 1 \mathrm{H}-2$ furan) $(\mathrm{J}=2.10 \mathrm{~Hz}), \delta 9.73(\mathrm{~s}, 1 \mathrm{H}, \mathrm{H}-3 \mathrm{Chromon})$ and $\delta 10.10(\mathrm{~s}, 1 \mathrm{H}, \mathrm{OH})$ (keto enol form).

\section{2-Amino-6-[6-hydroxy-4-methoxybenzofuran-5-yl]-4-styrylpyridin-3- carbonitrile (5)}

A mixture of compound 2 (3.2 gm, $0.01 \mathrm{~mol})$ malononitrile $(0.66 \mathrm{gm}, 0.01 \mathrm{~mol})$ and ammonium acetate $(0.77 \mathrm{gm}, 0.02 \mathrm{~mol})$ was fused at $100^{\circ} \mathrm{C}$ for 1 hour, then allowed to cool and poured into ethanol. The solid product was collected and crystallized from ethanol to give $\mathbf{5}$ (Table 1).

\section{Ethyl 2-Amino-6-[6-hydroxy-4-methoxybenzofuran-5-yl]-4-styryl-pyridin-3- carboxylate (6)}

A mixture of compound $2(3.2 \mathrm{gm}, 0.01 \mathrm{~mol})$ ethylcyano acetate $(0.01 \mathrm{~mol})$ and a few drops of ammonium acetate in ethanol $(30 \mathrm{ml})$ was refluxed for 3 hours, then allowed to cool. The solid product was collected and crystallized from n-hexane to give 6 Table (1). 


\section{5-(4,5-dihydro-1-phenyl-5-styryl-1H-pyrazol-3-yl)-4-methoxybenzo-furan-6-ol (7)}

A solution of 2 (3.2 gm, $0.01 \mathrm{~mol})$ and phenyl hydrazine (0.01 mole) in ethanol $(30 \mathrm{ml})$ was refluxed for 4 hours. The solid so obtained was crystallized from ethanol to give 7 Table (1). ${ }^{1} \mathrm{H}$ NMR $\left(\mathrm{CDCl}_{3}\right): \delta 2.43$ (d, 2H, pyrazol moiety), $\delta 4.24$ $\left(1 \mathrm{~s}, 3 \mathrm{H}, \mathrm{OCH}_{3}\right), 6.79-7.47(\mathrm{~m}, 16 \mathrm{H}, \mathrm{Ar}-\mathrm{H}+2=\mathrm{CH}+\mathrm{CH}$ of pyrazol $+\mathrm{H}-3, \mathrm{H}-2$ furan) and $\delta 10.92(\mathrm{br}, 1 \mathrm{H}, \mathrm{OH})$.

\section{5-(2-amino-4-styrylpyrimidin-4-yl)-4-methoxybenzofuran-6-ol (8)}

A mixture of 2 (3.2 gm, $0.01 \mathrm{~mol})$ guanidine hydrochloride $(0.59 \mathrm{gm}, 0.01 \mathrm{~mol})$ and potassium hydroxide $(0.5 \mathrm{gm})$ in ethanol $(50 \mathrm{ml})$ was refluxed 4 hours, then allowed to cool. The solid product was collected and crystallized from ethanol to produce $\mathbf{8}$ (Table 1).

\section{N-formyl-N-[4-(6-hydroxy-4-methoxybenzofuran-5-yl)-6-styryl-pyrimidin-2- yl]formamide (10)}

To a solution of $\mathbf{8}(3.59 \mathrm{gm}, 0.01 \mathrm{~mol})$ in $30 \mathrm{ml}$ of dry N,N-di-methylformamide, phosphorus oxychloride $(0.02 \mathrm{~mol})$ was added under stirring for about $30 \mathrm{~min}$. in an ice bath then the solution was poured into $200 \mathrm{ml} \mathrm{H}_{2} \mathrm{O}$. The solid product obtained was collected and recrystallized from ethanol to give 10 (Table 1). MS (10) $415\left(\mathrm{M}^{+}\right.$, $3.4 \%$ ) with a base peak at 283, and the following observed peaks at $345(8.1 \%) 327$ (3.07\%), 309 (45.9\%), $269(2.84 \%) 141(6.12 \%)$ and $133(54.8 \%)$.

\section{2-amino-4-(4-flurophenyl)-8-[6-hydroxy-4-methoxybenzofuran-5-yl]-6-styryl 6H-pyrimido[1,2-a]pyrimidin-3-carbonitrile (12)}

A mixture of 8 (3.2 gm, 0.01mole), p-flurobenzylidene malo-nonitrile (1.72 gm, $0.01 \mathrm{~mole})$ and piperidine $(0.5 \mathrm{ml})$ in ethanol $(30 \mathrm{ml})$ was refluxed for 3 hours, then allowed to cool. The solid product was collected and crystallized from ethanol to give 12, Table (1). Ms (12) showed a molecular ion $\mathrm{M}^{+}$at $\mathrm{m} / \mathrm{e}$ fragment $442\left[\mathrm{M}^{+}-89\right.$ (ph-C $\equiv), 57 \%$ ), with a base peak at 109 and the following peaks at $313(19 \%), 269$ (41.3\%), 230 (19\%), $190(23 \%)$ and 90 (38.1\%). 
Table (1): Characteristics data for the prepared compounds

\begin{tabular}{|c|c|c|c|c|c|c|}
\hline \multirow{2}{*}{$\begin{array}{l}\text { Comp. } \\
\text { No }\end{array}$} & \multirow{2}{*}{$\begin{array}{l}\text { M.P. } \\
{\left[{ }^{\circ} \mathrm{C}\right]}\end{array}$} & \multirow{2}{*}{$\begin{array}{c}\text { Yield } \\
\%\end{array}$} & \multirow{2}{*}{$\begin{array}{l}\text { Mol. formula } \\
\quad \text { (M. wt) }\end{array}$} & \multicolumn{3}{|c|}{$\begin{array}{l}\text { Elemental analysis } \\
\text { Calcd./found }\end{array}$} \\
\hline & & & & $\mathrm{C}$ & $\mathrm{H}$ & $\mathrm{N}$ \\
\hline 2 & $100-2$ & 87 & $\begin{array}{l}\mathrm{C}_{20} \mathrm{H}_{16} \mathrm{O}_{4} \\
(320.344)\end{array}$ & $\begin{array}{l}74.98 \\
74.99\end{array}$ & $\begin{array}{l}5.03 \\
5.04\end{array}$ & \\
\hline 3 & $318-20$ & 97 & $\begin{array}{c}\mathrm{C}_{27} \mathrm{H}_{22} \mathrm{ClNO}_{4} \\
(459.929)\end{array}$ & $\begin{array}{l}70.51 \\
70.52\end{array}$ & $\begin{array}{l}4.82 \\
4.83\end{array}$ & $\begin{array}{l}3.04 \\
3.05\end{array}$ \\
\hline 4 & $90-92$ & 62 & $\begin{array}{l}\mathrm{C}_{20} \mathrm{H}_{16} \mathrm{O}_{4} \\
(320.344)\end{array}$ & $\begin{array}{l}74.98 \\
74.97\end{array}$ & $\begin{array}{l}5.03 \\
5.02\end{array}$ & \\
\hline 5 & $98-100$ & 82 & $\begin{array}{c}\mathrm{C}_{23} \mathrm{H}_{17} \mathrm{~N}_{3} \mathrm{O}_{3} \\
(383.407)\end{array}$ & $\begin{array}{l}72.05 \\
72.11\end{array}$ & $\begin{array}{l}4.46 \\
4.51\end{array}$ & $\begin{array}{l}10.95 \\
11.10\end{array}$ \\
\hline 6 & $82-4$ & 46 & $\begin{array}{c}\mathrm{C}_{25} \mathrm{H}_{22} \mathrm{~N}_{2} \mathrm{O}_{5} \\
(430.46)\end{array}$ & $\begin{array}{l}69.75 \\
69.76 \\
\end{array}$ & $\begin{array}{l}5.15 \\
5.16\end{array}$ & $\begin{array}{l}6.50 \\
6.51 \\
\end{array}$ \\
\hline 7 & $73-5$ & 77 & $\begin{array}{c}\mathrm{C}_{26} \mathrm{H}_{22} \mathrm{~N}_{2} \mathrm{O}_{3} \\
(410.473)\end{array}$ & $\begin{array}{l}76.07 \\
76.08\end{array}$ & $\begin{array}{l}5.40 \\
5.42\end{array}$ & $\begin{array}{l}6.82 \\
6.84 \\
\end{array}$ \\
\hline 8 & 100-101 & 75 & $\begin{array}{l}\mathrm{C}_{21} \mathrm{H}_{17} \mathrm{~N}_{3} \mathrm{O}_{3} \\
(359.385)\end{array}$ & $\begin{array}{l}70.18 \\
70.15\end{array}$ & $\begin{array}{l}4.76 \\
4.78\end{array}$ & $\begin{array}{l}11.69 \\
11.67\end{array}$ \\
\hline 10 & $148-50$ & 93 & $\begin{array}{c}\mathrm{C}_{23} \mathrm{H}_{17} \mathrm{~N}_{3} \mathrm{O}_{5} \\
(415.405)\end{array}$ & $\begin{array}{l}66.50 \\
66.52\end{array}$ & $\begin{array}{l}4.12 \\
4.13 \\
\end{array}$ & $\begin{array}{l}10.11 \\
10.12\end{array}$ \\
\hline 12 & $114-116$ & 80 & $\begin{array}{c}\mathrm{C}_{31} \mathrm{H}_{22} \mathrm{FN}_{5} \mathrm{O}_{3} \\
(531.547)\end{array}$ & $\begin{array}{l}70.04 \\
70.05\end{array}$ & $\begin{array}{l}4.17 \\
4.21\end{array}$ & $\begin{array}{l}13.17 \\
13.18\end{array}$ \\
\hline
\end{tabular}

Table (2): Antimicrobial activity of some new benzofuran derivatives.

\begin{tabular}{|c|c|c|c|c|c|c|c|c|c|c|c|c|}
\hline \multirow[b]{3}{*}{ Organism } & \multicolumn{12}{|c|}{ Mean* of zone diameter, nearest whole mm. } \\
\hline & \multicolumn{4}{|c|}{ Gram-positive bacteria } & \multicolumn{4}{|c|}{ Gram-negative bacteria } & \multicolumn{4}{|c|}{ Fungi** } \\
\hline & \multicolumn{2}{|c|}{\begin{tabular}{|c|}
$\begin{array}{c}\text { Staphylococcus } \\
\text { aureus } \\
\text { (ATCC } \\
25923)\end{array}$ \\
\end{tabular}} & \multicolumn{2}{|c|}{\begin{tabular}{|c|} 
Streptococcus \\
Pyogenes \\
(ATCC \\
$19615)$ \\
\end{tabular}} & \multicolumn{2}{|c|}{$\begin{array}{l}\text { Pseudomonas } \\
\text { phaseolicola } \\
\text { (GSPB 2828) }\end{array}$} & \multicolumn{2}{|c|}{$\begin{array}{c}\text { Pseudomonas } \\
\text { fluorescens } \\
\text { (S 97) }\end{array}$} & \multicolumn{2}{|c|}{$\begin{array}{l}\text { Fusarium } \\
\text { oxysporum }\end{array}$} & \multicolumn{2}{|c|}{$\begin{array}{l}\text { Aspergillus } \\
\text { fumigatus }\end{array}$} \\
\hline \multirow{2}{*}{ Consent } & 1 & 2 & 1 & 2 & 1 & 2 & 1 & 2 & 1 & 2 & 1 & 2 \\
\hline & \begin{tabular}{c|}
2 \\
$\mathrm{mg} / \mathrm{ml}$
\end{tabular} & \begin{tabular}{c|}
1 \\
$\mathrm{mg} / \mathrm{ml}$
\end{tabular} & \begin{tabular}{c|}
2 \\
$\mathrm{mg} / \mathrm{ml}$
\end{tabular} & $\begin{array}{c}1 \\
\mathrm{mg} / \mathrm{ml}\end{array}$ & $\begin{array}{c}2 \\
\mathrm{mg} / \mathrm{ml}\end{array}$ & \begin{tabular}{c|}
1 \\
$\mathrm{mg} / \mathrm{ml}$
\end{tabular} & $\begin{array}{c}2 \\
\mathrm{mg} / \mathrm{ml}\end{array}$ & $\begin{array}{c}1 \\
\mathrm{mg} / \mathrm{ml}\end{array}$ & $\begin{array}{c}2 \\
\mathrm{mg} / \mathrm{ml}\end{array}$ & $\begin{array}{c}1 \\
\mathrm{mg} / \mathrm{ml}\end{array}$ & $\begin{array}{c}2 \\
\mathrm{mg} / \mathrm{ml}\end{array}$ & $\begin{array}{c}1 \\
\mathrm{mg} / \mathrm{ml}\end{array}$ \\
\hline 2 & $14, \mathrm{~L}$ & $9, \mathrm{~L}$ & $17, \mathrm{I}$ & $10, \mathrm{I}$ & $6, \mathrm{~L}$ & $4, \mathrm{~L}$ & - & - & $5, \mathrm{~L}$ & $3, \mathrm{~L}$ & - & - \\
\hline 4 & - & - & - & - & $7, \mathrm{~L}$ & $4, \mathrm{~L}$ & - & - & - & - & - & - \\
\hline 5 & - & - & - & - & - & - & - & - & $34, \mathrm{H}$ & $23, \mathrm{H}$ & $29, \mathrm{H}$ & $17, \mathrm{H}$ \\
\hline 7 & $15, \mathrm{~L}$ & $9, \mathrm{~L}$ & $16, \mathrm{I}$ & $9, \mathrm{~L}$ & $9, \mathrm{~L}$ & $5, \mathrm{~L}$ & - & - & - & - & - & - \\
\hline 8 & - & - & - & - & $18, \mathrm{I}$ & $10, \mathrm{I}$ & $16, \mathrm{I}$ & $7, \mathrm{~L}$ & $6, \mathrm{~L}$ & $3, \mathrm{~L}$ & - & - \\
\hline 10 & $32, \mathrm{H}$ & $21, \mathrm{H}$ & $20, I$ & $12, \mathrm{I}$ & $23, \mathrm{H}$ & $19, \mathrm{H}$ & $27, \mathrm{H}$ & $24, \mathrm{H}$ & - & - & - & - \\
\hline Control & 42 & 28 & 38 & 30 & 36 & 25 & 38 & 30 & 40 & 28 & 40 & 31 \\
\hline \multicolumn{13}{|c|}{$*=$ Calculate from 3 values $* * \quad=$ Identified depending on morphological and microscopical characters } \\
\hline
\end{tabular}




\section{References}

1. O. H. HISHMAT AND J. A. A. MICKY, Proc. Egypt. Acad. Sci., 38, 123 (1988).

2. N. M. SALEH, N. M. SHMEISS, S. A. MOHAMED, M. M. SALEM, AND J. A. A. MICKY, Al-Azhar Bull. Sci., 10 (2), 625-639 (1999).

3. O. H. HISHMAT, M. Y. ZOHAIR AND J. A. A. MICKY, Z. Natureforch, 38b, 1690 (1983).

4. J. A. A. MICKY, Al-Azhar Bull. Sci, 6(2) 1127 (1995).

5. J. A. A. MICKY AND S. A. M. ZAHKOUG, Natural product Science, 3(1), 273 (1997).

6. L. F. WIGGINS, J. W. JAMES AND M. W. GITTOS, Chem. Abstract., 84, 190749 (1976).

7. J. K. PANKA, K. VIJAI, T. K. GUPTA AND K. SHANKER, J. Indian Chem. Soc., 68, 422 (1991).

8. R. C. GUPTA, S. AHMED, M. B. GUTA, K. P. BHARGANA, K. KISHOR AND K. SHANKER, Indian Drugs, 17, 244 (1980).

9. O. H. HISHMAT, N. M. A. EL-EBRASHI, S. S. EL-NAKKADY AND M. T. ELKIRSH, Egypt. J. Pharm. Sci. 27 (1-4), 1, (1986).

10. R. C. FORT. AT AND P. VON R. SCHIEVER, Chem. Res., 64, 277 (1964).

11. A. C. HOLLINSHEAD AND P. K. SMITH, J. Pharmacol. Exp. Ther., 123, 54 (1968).

12. B. Melander, Antibiot. Chemotherapy, 10, 34 (1960).

13. J. R. CLARKE AND A. ROBERTSON; J. Chem. Soc., 302 (1949).

14. Z. H. KHALIL, A. S. YANNI, A. ABD EL-HAFEZ, A. A. KHALAF, J. Indian Chem. Soc. 67, 821 (1990).

15. A. W. BAUER, W. W. M. KIRBY, J. C. SHERRIS AND M., TURCK, Antibiotic Susceptibility testing by a standardized single disc method., Am. J. Clin. Pathol., 45, 493-496 (1966). 
تشييد بعض مشتقات البنزوفيوران الجديدة المتوقع لها نشاط بيولوجى

$$
\text { سعدية على حسين }
$$

$$
\text { كلية العلوم (بنات)-جامعة الأزهر - مدينة نصر - القاهرة - مصر }
$$

بمعالجـة 1-6 [-هيدروكسـى -4-ميثوكسـى بنزوفيـوران -5-يل][ايثـانون مــع

السنامالدهيا نتج 1-6-6يدروكسى -4-ميثوكسى بنزوفيوران -5-يـل]-5-فينيل بنتا4،2-دايين -1-اون الأى يتفاعل مع 4-كلوروانيلين والقورمالين ليعطى مثتقا من قواعد مـانش بينمـا بتفاعلهه مـع 20\% مسن حمـ الكبريتيـك المركز والمالونونيتريـل والايثيـل سيانواسيتات والفينيل هيدرازين والجواندين هيدركلوريد نتج فيورو[3,2-gكرومون -5اون ، البيريدين، البيرازول والبيريميدين المقابل. وعند تفاعل البيريميدين مع ثالث اوكسى كلوريـد القوسـفور ويـارا فلـورو بنزيليـلين مالونونيتريـل امكن الحصـول على مشـتقات الفورميل ويبريميدوييريميدين.

وقد تم اثبات التركيب البنائى للمركبات الجديدة بواسطة طيف الاشعة الحمراء و الرنيين النووى المغناطيسى وطيف الكتية. كمـا تـم اختبـار النشـاط البيولـوجى لبعض المركبـات المحضـرة حديثا ووجــ ان لبعضها نشاطا ملحوظا. 